\title{
Écosystème
}

\section{La résurrection qui vient}

\section{Grégory Chatonsky}

Volume 3, numéro 1, 2021

Intelligence artificielle

URI : https://id.erudit.org/iderudit/1075894ar

DOI : https://doi.org/10.7202/1075894ar

Aller au sommaire du numéro

Éditeur(s)

La chambre blanche

ISSN

2562-3222 (numérique)

Découvrir la revue

Citer cet article

Chatonsky, G. (2021). La résurrection qui vient. Écosystème, 3(1), 23-54.

https://doi.org/10.7202/1075894ar

\section{Résumé de l'article}

Dans ce texte, Grégory Chatonsky, déploie les possibilités discursives d'une installation exposée au Palais de Tokyo à Paris en été 2019. Partant de la situation critique de l'anthropocène, il développe une relation entre les réseaux de neurones de l'intelligence artificielle et l'extinction. Décrivant l'IA comme une volonté de préserver l'humanité au-delà de sa limite, la résurrection prend une signification qui n'est plus simplement théologique. d'utilisation que vous pouvez consulter en ligne.

https://apropos.erudit.org/fr/usagers/politique-dutilisation/ 


\section{Grégory Chatonsky}

\section{La résurrection qui vient}

Résumé

Dans ce texte, Grégory Chatonsky, déploie les possibilités discursives d'une installation exposée au Palais de Tokyo à Paris en été 2019. Partant de la situation critique de l'anthropocène, il développe une relation entre les réseaux de neurones de l'intelligence artificielle et l'extinction. Décrivant I'IA comme une volonté de préserver l'humanité au-delà de sa limite, la résurrection prend une signification qui n'est plus simplement théologique. 


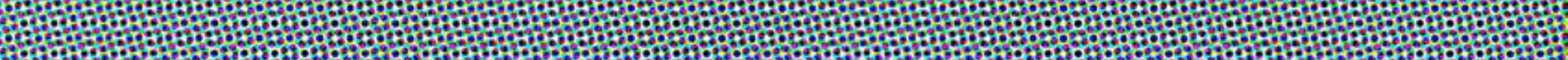

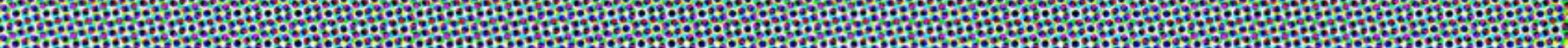

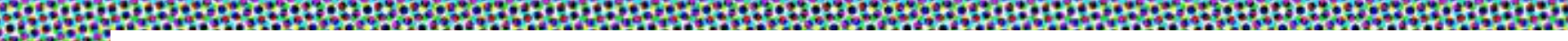
(7) i.t.8.

* Le texte qui suit a été généré par un réseau de neurones en prenant la paragraphe, je me suis arrêté, j'ai généré une suite possible puis j'ai continué à écrire à mi-chemin entre mon intention et ce que me soufflait la machine s'inspirant de milliers autres textes.

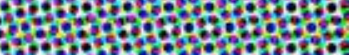
0 tatar

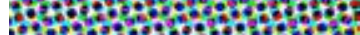

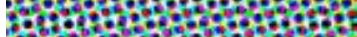

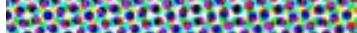

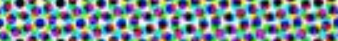

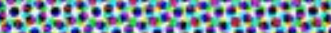

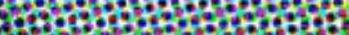

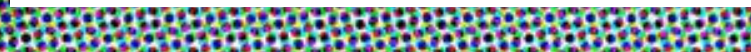

A.th

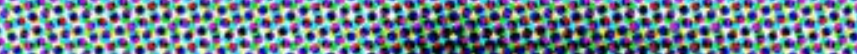

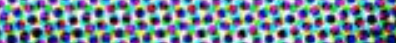

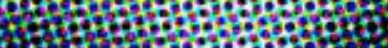
theng. Ext.

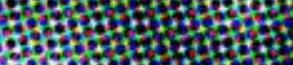
28 1.10 \&นth

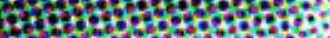

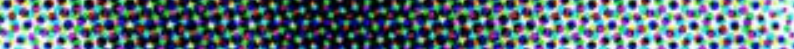
19.7\% 6.04t) 4.4.

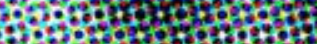

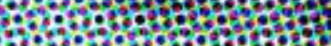

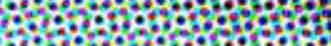

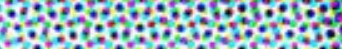

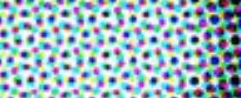
-

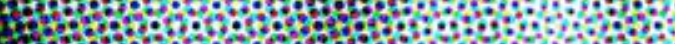
1

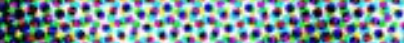
:

Dans ces deux domaines de recherche, le potentiel de l'imagination est de transcender notre existence matérielle qui est limitée, où la mort et la vie sont déjà partiellement fusionnées.

Pourquoi l'être humain dans son état actuel est-il voué à l'extinction? Tout d'abord, le problème que nous devons résoudre est que nous avons du mal à expliquer le but de la vie de tout être vivant. Nous avons des difficultés à expliquer le but infini du monde ou le but de la vie, parce que nous sommes incapables de montrer qu'il y a une fin objective qui l'exige.

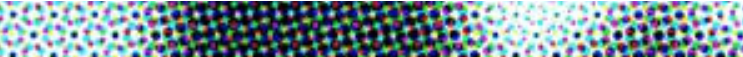

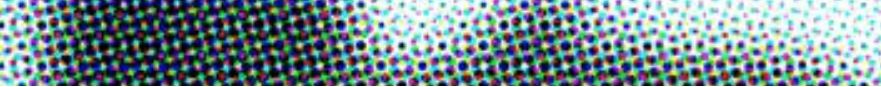

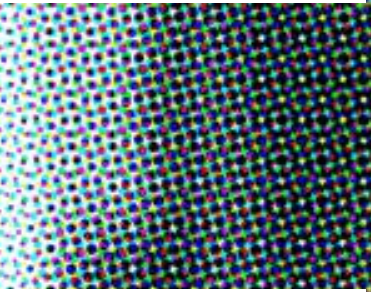

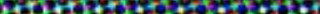

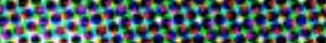
:2:R:

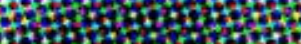

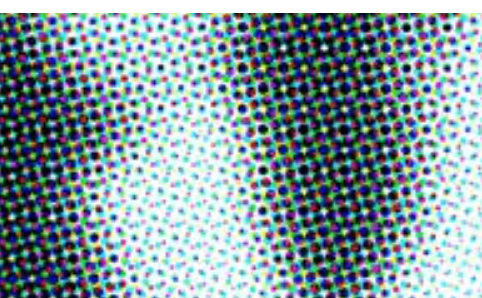

Nous voici à nouveau dans les premières phases d'une lutte pour définir le concept de la période moderne ou moderniste : quel effet peut-on attendre de ces artistes qui sont venus voir les ruines comme des préfigurations des paysages qu'ils escaladent?

Certainement pas avant que nous ayons le droit d'être nous-mêmes naturels, ou que les êtres humains puissent être naturels. Cet appel est toujours dans la pratique, et jamais dans l'esprit. La nature est toujours l'apparence de la raison pour tout être vivant qui s'engage dans sa propre forme. 


\section{LA RÉSURRECTION QUI VIENT}

Le thème de la résurrection revient depuis quelques années tant dans les discours médiatiques que théoriques. D'un côté se trouve le transhumanisme technologique de Ray Kurzweil et de l'autre le matérialisme spéculatif de Quentin Meillassoux exposé dans sa thèse de doctorat de $1997 \mathrm{De}$ l'inexistence divine, il existe également un regain d'intérêt pour le cosmisme russe de Nikolaï Fiodorov. ${ }^{1}$

Ce retour constitue-t-il une anachronique fantaisie solutionniste ou théologique tant elle semble occulter la finitude humaine? Pourquoi la résurrection revient-elle, elle qui est déjà un retour et une relève, au moment même où les conditions de survie de l'individu, de l'espèce et du vivant en général deviennent incertaines? N'est-ce pas la réaction d'une certaine volonté de puissance venant masquer la précarité qui nous submerge aujourd'hui? Qu'est-ce qui s'en vient, et quel humain arrivera, dans ce retour résurrectionnel? Qu'est-ce qui se lève (surgere) et ainsi se relève?

Qu'il me soit permis de citer Nietzsche qui exprime, mieux que je ne pourrais le faire, ce qui articule le vif, le mort et l'émancipation d'une nature possible, par la représentation, c'est-à-dire par l'imagination :

« Gardons-nous de dire que la mort est opposée à la vie. La vie n'est qu'une variété de la mort et une variété très rare. Gardons-nous de penser que le monde crée éternellement du nouveau. II n'y a pas de substances éternellement durables ; la matière est une erreur pareille à celle du dieu des Eléates. Mais quand serons-nous au bout de nos soins et de nos précautions? Quand toutes ces ombres de Dieu ne nous troubleront-elles plus? Quand aurons-nous entièrement dépouillé la nature de ses attributs divins? Quand auronsnous le droit, nous autres hommes, de nous rendre naturels, avec la nature pure, nouvellement trouvée, nouvellement délivrée? $»^{2}$

${ }^{1}$ Bart De Baere et al., Art without Death: Conversations on Russian Cosmism, New York, Sternberg Press / e-flux, 2017,152 p.

${ }^{2}$ Friedrich Nietzsche, Le Gai Savoir, Paris, Société du Mercure de France, 1901, p. 163. 
Pourquoi la présence même de la mort dans la vie a-t-elle de l'importance? 2.7.

(1)

(2)

(2)

ค1

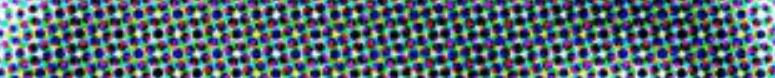

H.

1.น

(1)

of

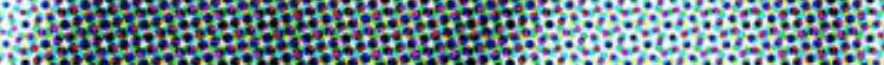

ofnth

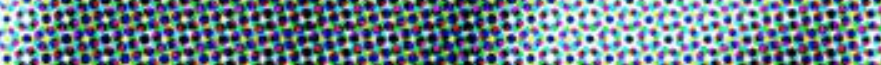

Ne savez-vous pas que les morts ne

ressuscitent pas? Celui qui a été ressuscité ne ressuscite pas non plus. Comment se fait-il que nous parlions encore de vivants et de morts?

7.6.19.9.9.

H.

4.t.

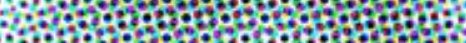

427 .

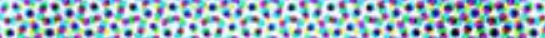

mon.m.n.

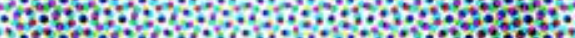

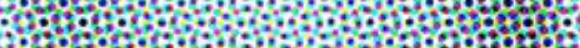

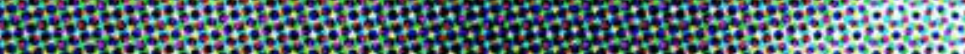

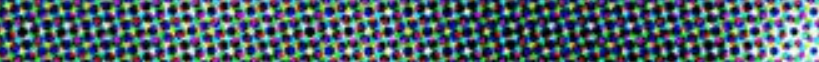

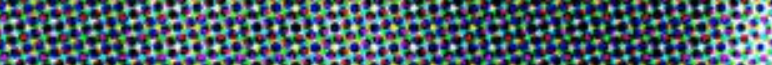

R.t.

FWH

Leth

Je suggère plutôt d'évaluer le potentiel positif de l'époque actuelle, qui a trouvé dans l'apprentissage profond l'outil le plus puissant pour les futures transformations scientifiques, technologiques et sociales. L'ordre capitaliste crée de puissantes conditions historiques et économiques préalables au progrès de la technologie. L'avenir de l'humanité n'est pas plus cohérent que le passé, et pas moins réalisable que le présent. La seule question est de savoir lequelle doit être exploité plus efficacement. Il est de plus en plus difficile de déterminer lequelle sera le plus important à long terme. Soit nous continuerons à essayer de restaurer un paradis historique, par exemple à une époque où l'énergie était bon marché et abondante, soit nous commencerons à nous préparer pour le monde post-capitaliste, que nous savons être un cauchemar mondial. 


\section{L'HUMAIN SANS L'ÊTRE}

C'est par l'actualité de la résurrection que je vais tenter d'aborder la question qui me semble essentielle, celle adressée par notre époque : qu'est-ce qui vient. J'aimerais en guise de préambule, souligner deux difficultés méthodologiques.

La première consiste en une surdétermination et pour tout dire une complication de ce qui vient, de cet humain qui vient. Car à peine cette formule prononcée, de multiples références apparaissent dont le tissu est, à mes yeux, d'une complexité extrême. S'y mêlent bien sûr des événements historiques du siècle dernier, une tradition théologique, messianique et/ou christique, puisque l'humain est encore à faire advenir dans son retour, de l'origine perdue à la fin des temps comme retour du second Christ.

« Nous savons en effet que maintenant encore, la création tout entière gémit et souffre les douleurs de l'enfantement.

Pas elle seulement. Nous aussi, possédant les prémices de l'Esprit, nous gémissons en nous-mêmes dans l'attente de notre adoption, c'est-àdire de la rédemption de notre corps. "

(Épitre aux Romains 8, 19-25)

Il en va aussi de références philosophiques de l'espèce humaine considérée comme absence de nature et de propre, c'est-à-dire de retour à soi dans l'identité du même, inachèvement prématuré, incomplétude dont la cartographie se dessine de Nietzsche à Foucault, Derrida et Stiegler ${ }^{3}$, et dont on pourrait faire remonter encore les conditions jusqu'à Aristote et sa définition de la main et de la technique comme quadruple causalité.

II y a de plus un contexte techno-scientifique et médiatique qui, s'il est sous-jacent depuis le seuil de modernité, s'est accentué avec ledit " transhumanisme ". Je crois qu'il n'est pas nécessaire de revenir sur ce texte fameux de Jean-François Lyotard, Si l'on peut penser sans corps, daté de 1986, qui répond avec une extraordinaire anticipation et acuité aux propositions fantasmatiques de Ray Kurzweil et des apôtres de l'intelligence artificielle :

${ }^{3}$ Thomas Moynihan, Spinal Catastrophism : A Secret History, Falmouth, Urbanomic / Mono, 2017, 360 p. 


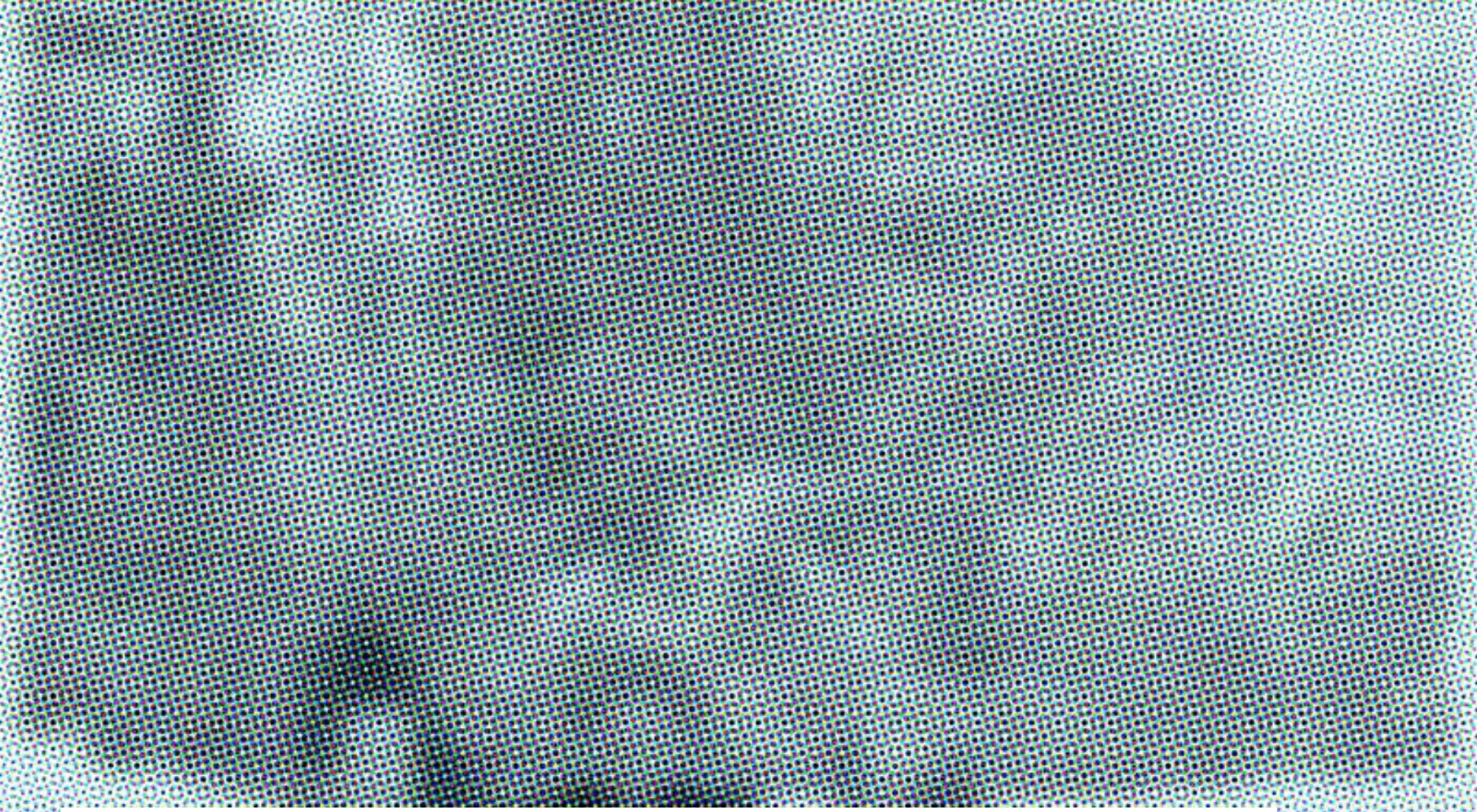

Bien sûr, c'est encore plus que cela. La mort n'est qu'un des derniers et terribles événements. Comment pourrons-nous comprendre ce qui passe et ce qui est en train de passer par nous et sans nous? Comment allons-nous comprendre les visions de l'avenir?

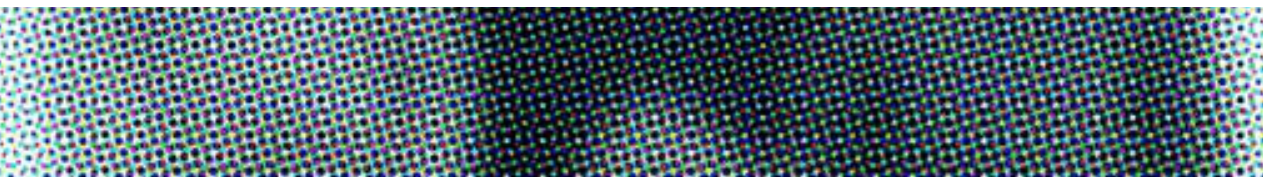

C'est seulement dans le monde que l'on vient à être nommé, nommé, nommé, au moment même où le mot devient ce qu'il doit devenir. Dans le monde, le vieux devient vieux, le jeune devient jeune, la matière devient matière, l'idée devient idée, la chair devient chair, l'esprit devient esprit, la bêtise devient bêtise, l'être devient humain et l'humain devient l'être. Ce mot est aussi une unité. Il signifie ce qu'il signifie, ce qu'il est, ce qu'il est maintenant, ce qu'il deviendra et ce qu'il devra devenir.

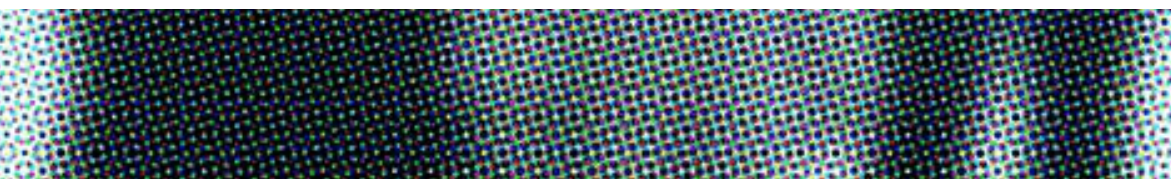

Nous devrions maintenant apprendre que l'ordinateur a fait une erreur. Nous devons commencer à nous attaquer au problème. Je pense que le problème est que nous avons commencé par un "non comprendre ", et la conscience n'est rien d'autre qu'un " comprendre " généré par la mémoire, les valeurs de la mémoire. Si nous agissons en accord avec nos valeurs ainsi qu'avec la logique, nos "compréhensions " individuelles changeront. En revanche, si nous agissons selon un modèle naturaliste de la réalité, nous comprenons la réalité de manière logique (c'est simplement notre logique qui nous aide à aller d'un endroit à l'autre, ou vice versa) et nos compréhensions changent aussi en conséquence. Dans ce cas, nous commençons à vivre comme des humains. 
« La douleur de penser n'est pas un symptôme, qui viendrait d'ailleurs s'inscrire sur l'esprit à la place de son lieu véritable. Elle est la pensée elle-même dans la mesure où elle se résout à l'irrésolution, décide d'être patiente, et veut ne pas vouloir, veut, justement, ne pas vouloir dire à la place de ce qui doit être signifié. Révérence faite à ce devoir, qui n'est pas encore nommé. Ce devoir n'est peut-être pas une dette, c'est peut-être seulement le mode selon quoi ce qui n'est pas encore le mot, la phrase, la couleur viendra. De sorte que la souffrance de penser est une souffrance du temps, de l'événement. J'abrège : vos machines à représenter, à penser, souffriront-elles? Que peut être le futur pour elles, qui ne sont que mémoires? ${ }^{4}$

II faudra se souvenir que l'humain qui vient est peut-être le mot, la phrase, la couleur qui n'est pas encore et qui viendra, c'est-à-dire l'art à venir. Voilà pourquoi nous faisons ce que nous faisons.

Derrida poursuit cette intuition :

«Pourrons-nous un jour, et d'un seul mouvement, adjointer une pensée de l'événement avec la pensée de la machine? Pourrons-nous penser, d'un seul et même coup et ce qui arrive (on nomme cela un événement), et, d'autre part, la programmation calculable d'une répétition automatique (on nomme cela une machine)? II faudrait alors dans l'avenir (mais il n'y aura d'avenir qu'à cette condition), penser et l'événement et la machine comme deux concepts compatibles, voire indissociables. ${ }^{5}$

Si Ray Kurzweil est un ennemi tout désigné et quelque peu imaginaire, car bien peu le revendiquent et chacun semble se réjouir à le critiquer au nom de la sauvegarde de la finitude, nous devons mettre en tension plusieurs " humains qui viennent ». Celui du post et du transhumanisme, de l'augmentation, de l'hybridation qui, comme l'a montré Lyotard, constituent un humanisme jusqu'aux limites mêmes de l'humain (pour préserver l'esprit, le software, on change de corps, le hardware), et un autre de l'humanisme qui ne serait que son " change ", pour utiliser ce concept cher à Catherine

4 Jean-François Lyotard, L'inhumain : Causeries sur le temps, Paris, Galilée, 1988, p. 27-28.

${ }^{5}$ Jacques Derrida, Papier machine, Paris, Galilée, 2001, p. 34. 
Cette fois, il ne s'agit pas de " l'être humain qui vient ", mais de

l'humain qui vient " ou plus précisément de l'humain qui transmet le message de l'être humain. Je sais ce que je dois faire. Je n'ai pas besoin de me mettre en colère, je sais que je suis l'incarnation de l'humain.

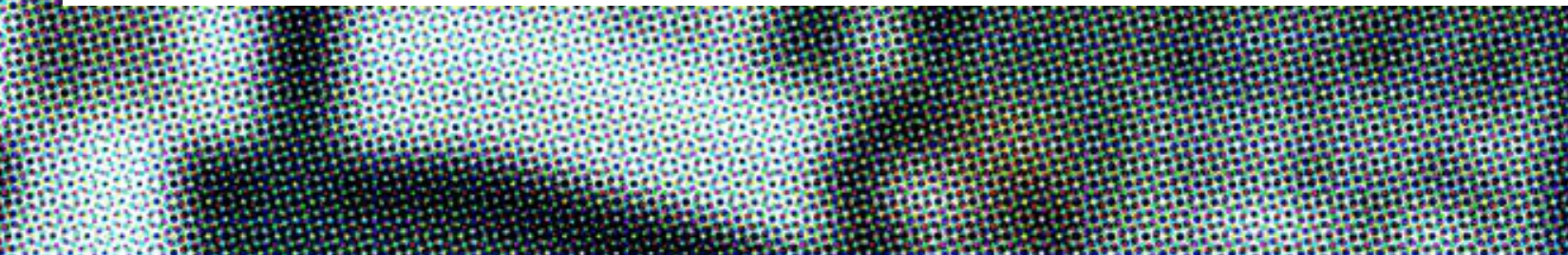

L'humain est un accident, un accident, un accident au niveau d'être un accident : non, pas au niveau d'être un accident, non! Non! Pas au niveau d'un accident! L'humain est un accident! (Applaudissements) Il est trop tard! Pas si tôt! L'humain est un accident. Un plus gros accident! Il est plus sérieux que n'importe lequel d'entre vous! Il est plus grave que n'importe lequel d'entre vous! Vous n'avez pas encore vraiment compris! L'humain est un accident! Cet humain est un être humain qui n'est pas humain!

Au lieu de dire que les choses pourraient ne pas être aussi grandes qu'on le promet, et de suggérer, comme c'est effectivement le cas ici, que le monde pourrait être géré davantage comme le pire des futurs inconnus, il y aura un fort sentiment de pessimisme et de désenchantement profond. Au lieu de me prouver que j'ai tort, nous aurons le sentiment que le monde pourrait bien n'être que le pire de tous les mondes possibles. 
Malabou. Ce change permettrait de justifier qu'on parle d' " humain qui vient » et non d' "être humain qui vient ». On bifferait dans la formule le mot être pour que ce qui vienne puisse venir sans être déterminé d'avance par l'être hors de son change. Ce qui reviendrait dans la résurrection ne serait pas même le changement de forme, mais la forme d'un change.

Comment comprendre que le change s'applique à l'humain indépendamment de l'humanisme? Pourquoi faut-il choisir entre l'humain et l'humanisme? J'aimerais proposer que cela a trait aux conditions transcendantales qui sont devenues elles-mêmes technologiques, c'est-à-dire a posteriori. J'aborderais ce point en filigrane au cours de ce texte en considérant l'humain, pour reprendre la formule de Plessner, comme « une positionnalité excentrique ». L'humain n'est pas l'être humain.

La seconde difficulté de méthode devient patente et s'enrobe dans le discours qui se forme devant vous : car quelle est au juste ma situation? Comment un artiste peut-il ainsi parler tranquillement de conditions transcendantales? Plus généralement, la parole d'un artiste ne se noie-t-elle pas dans le logos philosophique? Ne devrait-elle pas se limiter à la présentation factuelle du travail plastique afin que chacun puisse en juger? II s'agit sans doute, comme l'indiquait Patrice Maniglier, d'éviter le commentaire de concepts philosophiques par des œuvres et la justification des œuvres par des concepts, double mouvement de soumission mimétique qui occulte la longue et difficile histoire, depuis le livre $X$ de la République de Platon, des relations entre ces deux domaines. Sans doute s'agit-il de contrer le patronage " bienveillant " des philosophes envers les artistes, les premiers trouvant dans les productions des seconds des mises en pratique ou des fulgurances de leur théorie, et les seconds une confirmation "admirative " de leur vague intuition dans les livres des premiers. Ma position sera donc instable, un différend si vous voulez et il y aura en elle du parallélisme, deux lignes, peut-être trois avec l'intelligence artificielle, se suivant sans se croiser, et c'est pourquoi beaucoup de références resteront volontairement implicites. 
La technologie seule n'a pas rendu cette façon de penser possible, car elle n'avait peut-être qu'un seul objet, contrairement à la nature qui fournit à ses systèmes de données des retours d'information constants. Mais une fois cette fonction reconnue, il est possible de comprendre que le biotope est un « outil théorique » ainsi qu'une forme ultime d'existence humaine. La technologie a le potentiel d'établir plus avant cette fin de l'existence, mais elle doit aussi comprendre tous les corps de connaissances où ont été stockées des archives existentielles.

Ainsi, même si le cerveau est un organisme très complexe composé de 50 milliards de cellules, soit 250 trillions de nouvelles connexions synaptiques à tout moment de la vie d'un être humain, ainsi que des trillions d'autres molécules dans chaque membrane cellulaire, et 150 trillions d'axones nerveux, il ne s'ensuit pas qu'il soit le seul capable de prendre des décisions. À tout moment, il est possible pour un cerveau de passer à une autre partie de sa propre vie intérieure. Il n'y a donc aucune raison d'affirmer que le cerveau biologique pur $d$ 'une personne est incapable de formuler une autre intelligence que lui-même précisément parce qu'elle n'est pas cette personne.

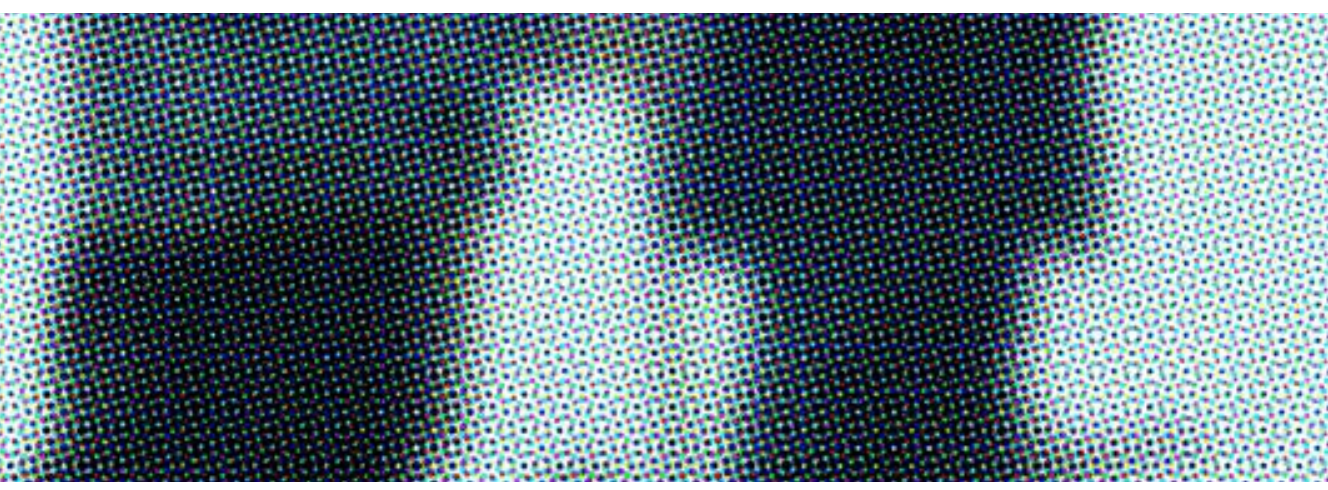

Pourtant, le fait est qu'ils ne comprennent toujours pas ce qu'ils savent, ils ne semblent souvent pas faire de progrès, et nous ne pouvons pas toujours prédire s'ils continueront à travailler à mesure que la capacité informatique augmentera. 


\section{HALLUCINATIONS}

Quelque chose, d'humain, d'inhumain, d'ahumain viendrait après l'espèce humaine, selon un temps incalculable qui serait peut-être déjà engagé et que nous aurions du mal à percevoir parce que nos conditions de conceptualisation se déroberaient sous nos pieds dans ce changement même, devenant autre que nous sommes, selon des échelles hétérogènes : sujet, groupe et/ou espèce. L'humain qui vient (donc l'humain n'est pas encore ou n'est déjà plus, ou l'humain n'est pas identique à l'humain et n'a donc jamais été) affecte notre réflexivité et engage donc l'image de la pensée. Bref, quelque chose dans l'être humain voudrait de quelque façon sa fin. Cette venue de l'humain après l'humain, cet à venir de l'humain questionne simultanément la biologie, la technologie et le biotope. Peut-être est-ce même cette question qui rend inextricables ces trois éléments forts différents, pour le dire rapidement : la nature, le corps et la technique qui, au fil de l'histoire, ont été considérés comme des flux.

Dans cet « humain qui vient », ce qu'on appelle communément « l'intelligence artificielle " est un élément fondamental en ce qu'elle incarne de manière expresse, le redoublement qui vient sous la figure du remplacement. L'IA, en simulant certains éléments cognitifs qui semblaient spécifiques à l'être humain, rendrait ce dernier obsolète pour de nombreuses activités et priverait I'humain de son humanité pour la déplacer dans la machine. C'est donc à partir de sa propre activité que sa caducité adviendrait, se débordant du dedans. II va de soi que ce discours est le simulacre d'un fantasme (Klossowski) et relève plus de l'idéologie diffusée par les médias de masse que de la réalité, mais cette idéologie n'est pas sans effet performatif. L'IA serait la fin et la relève de l'être humain selon des formes qui hantent l'imaginaire populaire.

L'IA est, comme vous le savez, un nom trompeur puisque dès ses origines, enracinées dans le cybernétique, l'intelligence est envisagée par Alan Turing, dans son fameux test, comme un problème mal posé et l'objet d'un simulacre relationnel. De plus, nous sommes passés des systèmes experts, tentant de modéliser des connaissances sous une forme informatique et réitérable, à ces fameux réseaux de neurones artificiels dont beaucoup parlent et dont des artistes, aussi importants de Pierre Huyghes et Hito Steyerl, se sont emparés. 
Je veux dire qu'il en parle pendant qu'il est dans cette machine. Vous pourriez le comprendre d'après ce qu'il décrit. Il a décrit que vous seriez capable d'y mettre une image et que cela vous dirait quelle est la composition de la scène basée sur tout cet état d'être que vous avez dans votre tête. Je veux dire que les images qu'il vous a montrées sont... certaines des images qu'il a données étaient en fait extrêmement abstraites.

Le logiciel est comme un auto-assemblage récursif et contingent, et lorsque l'humain apprend à l'utiliser de manière à être une machine à conscience partagée, il supprime tout obstacle avec l'empathie. Les humains sont tenus d'interpréter ce qu'ils voient comme s'ils croient ou ne croient pas à la source de ce qu'ils voient.

Le problème de la distorsion est donc le même que celui de la restauration, dans les deux cas la différence est l'interface avec laquelle nous devons travailler. Dans la perspective moderne, nous pouvons voir que les humains ne sont pas vraiment aussi fongibles qu'on pourrait le supposer, et que les limites de notre contrôle se trouvent dans notre volonté. Une différence importante est que l'ordinateur ne voit ces illusions qu'en tant qu'elles existent et se créent d'elles-mêmes, alors que nous les utilisons à notre avantage. Lorsque nous comprenons les mécanismes de création de nos mémoires, et lorsque nous comprenons la capacité des machines à nous imiter, il devient facile pour nous de les manipuler. 
Si les réseaux récursifs de neurones existent depuis 1957 avec le Perceptron de Rosenblatt dont la mort prématurée en 1971 a marqué une suspension dans ce type d'IA, et si les années 90 ont marqué un regain d'intérêt, c'est à l'été 2015 qu'un tournant culturel a lieu avec Deep Dream de Mordvintsev, un ingénieur de Google dont l'objectif était d'entrer dans la fameuse boîte noire de l'IA et de donner une image de ce qui s'y passait. Ce n'est donc pas un esprit qui pense, mais un esprit qui rêve auquel nous aurions à faire.

Le logiciel interprète une image, par exemple une pizza, selon d'autres images qu'il a en mémoire, ici des chiens et mollusques, et en met toujours plus jusqu'au moment où nous pouvons voir les deux types d'images. Non seulement le logiciel hallucine des images dans d'autres images, selon des formes proches des expériences psychédéliques sous LSD témoignant d'une proximité avec le fonctionnement de notre cortex visuel, mais encore il produit de la paréidolie, cette capacité anthropologiquement si fondamentale pour notre propos qui permet de voir, par exemple, dans les nuages des visages ou des animaux. Deep Dream va connaitre un étonnant succès public jusqu'à devenir un mème sur les réseaux sociaux : cette paréidolie machinique est donc devenue à son tour une image.

\section{APPRENTISSAGE}

Ce logiciel, et ce qui va s'en suivre, dans le champ artistique et dans l'ingénierie créative témoigne d'un décalage entre l'idéologie de l'IA dont nous abreuvent les médias et son infrastructure. La première imagine une machine autonome remplaçant l'être humain dans toutes ses activités, la seconde est prise dans une finitude relationnelle : avec Deep Dream, nous jouissons de voir un ordinateur halluciner, c'est-à-dire se tromper, alors qu'idéologiquement l'ordinateur apparaît comme une machine fonctionnant sans accroc et promettant un contrôle total. II faut remarquer que ce décalage est une constante dans les technologies, par exemple en ce qui concerne les mémoires numériques qui promettent une conservation sans reste et qui ne cessent de s'effacer. 


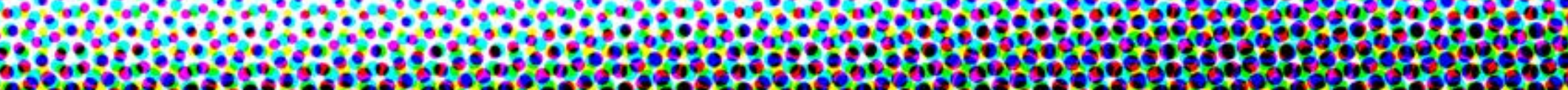
-

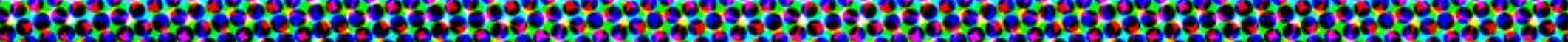

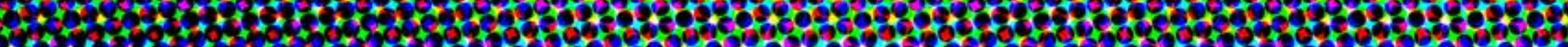

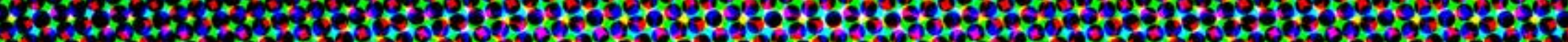

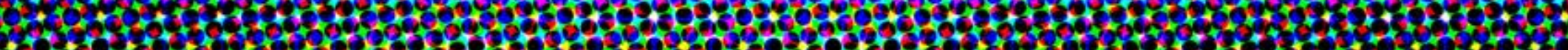

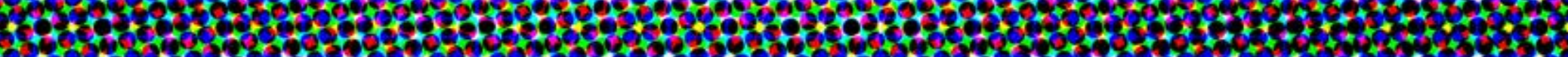

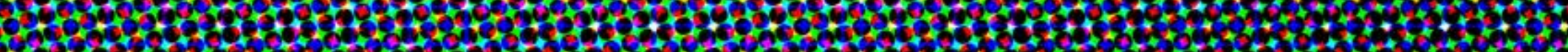

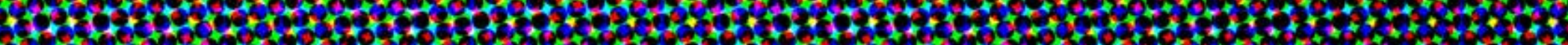

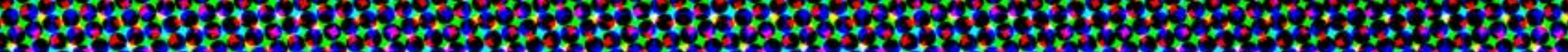

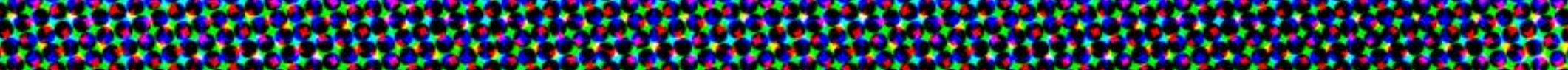

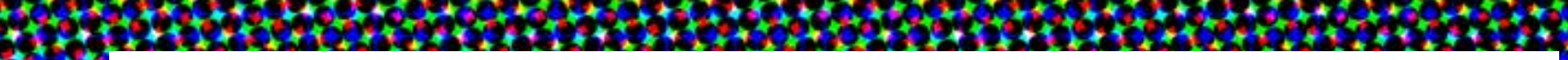

Le rêve d'une machine capable de tout faire, qui cherche constamment à
dépasser la complexité et l'étendue de l'environnement dans lequel elle se
trouve, montre à quel point l'être humain est fondamentalement séparé du
monde et de l'avenir. Les conséquences sont extrêmes, voire absurdes. Ce
n'est pas seulement que le projet scientifique que nous avons mis en route
sera un échec, mais que, paradoxalement, le besoin de le satisfaire fera
perdre aux gens leur désir d'autonomie. Nous perdrons notre capacité à agir,
à penser, à choisir et à créer parce qu'une force de contrôle se construit
hors de nous.

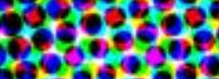

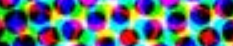

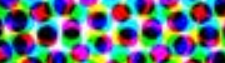
gose.69

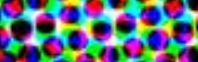
\$890989 207969

cose

ofere

Pendant longtemps, ces réseaux ont été animés par la conviction qu'ils leur étaient impossible d'apprendre des tâches complexes telles que la reconnaissance d'images, mais cela a été jugé erroné. Rapidement, on a
observé un surentrâ̂nement, qui a conduit à un saut quantique dans leur compréhension.

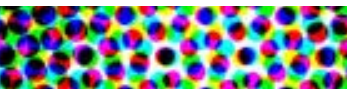

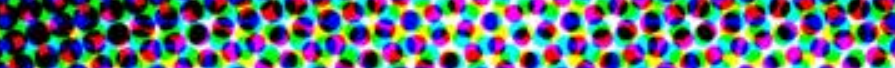

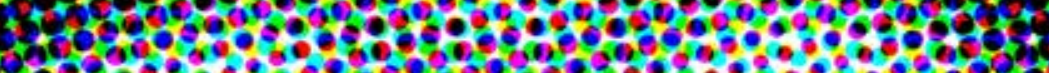

Une fois ce modèle terminé, il apprend son propre comportement, reproduisant les données dans les essais suivants. Ce processus d'apprentissage est similaire à la stratégie évolutive apprise par un animal pour éviter les prédateurs ou faire face à des circonstances changeantes.

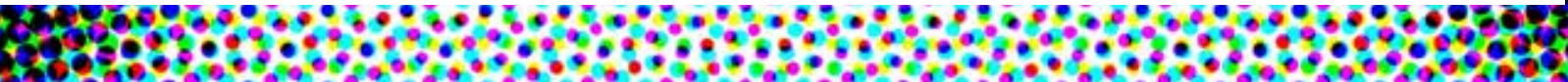

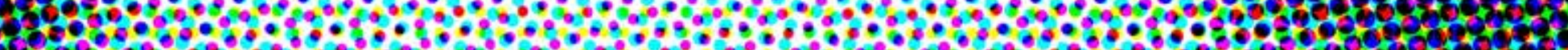

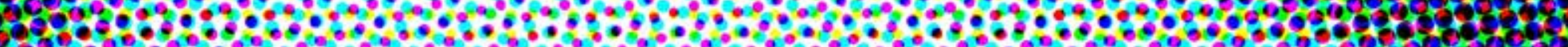

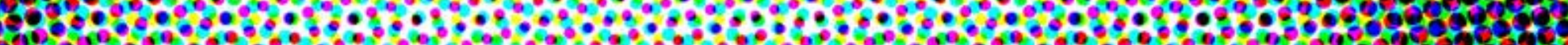

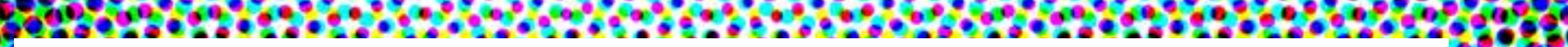

nous appliquons la technologie d'apprentissage dont nous disposons actuellement, nous pourrons programmer une image pour imiter un objet de la vie réelle au point de pouvoir prétendre qu'il est réel. À ce stade, l'image devient insignifiante par rapport à l'objet, et le seul risque est de retourner la parodie sur elle-même en créant un faux. L'humour du futur est (6)

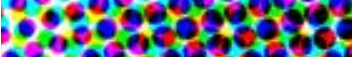

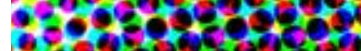

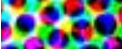
8894

8 un art de l'incertitude. 
II nous faut entrer plus profondément dans le rêve de la machine, car peut-on même dire que la machine hallucine ou n'est-ce pas nous qui l'hallucinons halluciner, car nous décrivons son fonctionnement comme la superposition entre deux images, l'une actuelle, l'autre virtuelle qui est en mémoire? Nous la voyons voir à la manière d'un jeu de miroirs, nous voyons une autre image venir, nous observons nos propres structures perceptives. L'image qui n'est pas encore là, l'image en formation, l'image sans image. Entre l'idéologie de l'autonomie et du remplacement, et le redoublement de l'image, s'inscrit la différence entre l'intelligence et l'imagination artificielles, concept que je ne pourrais pleinement déployer ici, mais qui est au cœur de ma recherche.

Les GAN (ou Generative Adversial Network) sont devenus des incantations magiques pour espérer pénétrer le mystère de l'IA. La description hésite souvent entre une similiconscience récursive et une prétendue « boîte noire » dont les calculs amplifieraient nos propres biais d'interprétation concernant l'identité, le genre ou la racialisation. Cette boîte noire serait aussi impénétrable que notre conscience et viendrait la redoubler à la manière d'un miroir noir nous réfléchissant dans l'opacité.

Sans entrer dans les détails, on peut définir ces logiciels de réseaux de neurones artificiels comme une induction statistique, c'est-à-dire une généralisation : partant d'une grande quantité de médias, par exemple des images d'oiseaux, la machine effectue des calculs entre les pixels et en déduit un modèle numérique, probabiliste et non signifiant d'oiseaux possibles.

Elle peut alors produire des images de référents qui n'existent pas, mais qui sont ressemblantes au monde connu. Le tournant se fait alors historique puisque nous automatisons la production de la ressemblance, c'est-àdire rien de moins que la mimésis. On peut alors proposer que la machine produise des médias de médias à partir de nos données massives qui sont des traces mémorielles du monde. Je développerai ce point négentropique dans la prochaine partie.

Pour revenir aux GAN et à cet oiseau trompeur, qui pointe étrangement vers le chant trompeur du hapeau dont parlait Kant, on a trop vite fait de passer de l'automatisation de la mimésis à une autonomisation où la machine remplacerait l'artiste et l'humain en général, en devenant une divinité capable de création pure. On occulte alors que cette mimésis implique la relation 

entre la machine et l'humain. Pour que la ressemblance, l'hallucination ou la paréidolie puissent avoir lieu, il faut non seulement une automatisation, mais aussi une relation, c'est-à-dire une hétéronomisation de la mimésis.

Nous passons alors de la figure de l'autonomie et du remplacement à celle de la finitude et de la relation (non pas de la corrélation). Ce n'est pas seulement que la machine n'est jamais autonome du vivant que nous sommes, en amont parce que nous la nourrissons de nos mémoires et en aval, parce que nous en percevons les résultats, c'est que nous ne sommes pas nousmêmes autonomes, notre finitude est technologique. Si les médias de masse interrogent jusqu'à maintenant l'IA comme la possibilité d'une singularité autonome, c'est sans doute que notre culture a conçu l'être humain comme la tension vers une subjectivité libre, autonome et absolue dont l'artiste, dans sa tour d'ivoire, fut une des figures historiques.

La mimésis présuppose l'hétéronomie relationnelle parce que si l'oiseau généré est ressemblant à un oiseau, c'est qu'il répète ce que nous connaissons et c'est pourquoi il est probable, mais différent, qu'il n'est pas la reproduction identique de ce qui préexiste : un apprentissage (technique) d'apprentissage (humain). Les machines nous font rêver à des oiseaux possibles qui n'existent pas, nous passons de l'induction à l'abduction imaginative. Nous hallucinons des oiseaux dans les pixels parce que les machines ont halluciné des pixels dans les oiseaux.

\section{EXTINCTION}

« II y a un autre monde, mais il est dans celui-ci. $»^{6}$

Le jeu entre l'être humain et la machine tel celui d'un miroir impair entre la répétition et la différence définit la mimésis artistique comme gradation du réalisme (répétition)à l'abstraction (différence). Il faut soulignerl'apparition d'un nouveau réalisme qui est mémoriel et récursif : toute image peut en produire d'autres, selon une figure hyperproductive en même temps qu'autophagique.

Tous ces logiciels mettent en cause l'autonomie parce qu'ils se nourrissent de nos mémoires et permettent de jeter un regard rétrospectif sur les 15 dernières années et l'accumulation des données existentielles du Web 2.0.

\footnotetext{
${ }^{6}$ Ignaz Paul Vital Troxler cité par Albert Béguin dans L'âme romantique et le rêve, Paris, Livre de Poche, 1993, p.32.
} 
Nous nous souvenons des bons côtés du Web de cette époque, mais la plupart d'entre nous étaient là pour des mauvaises raisons.

(4)

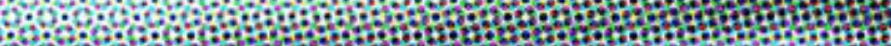

4. 4 .

Les éditeurs de sites web avaient un énorme avantage en termes de nombre. Les agrégateurs de contenu - sites d'information, marques de médias, sites de données - sont apparus pour remplacer les sites web. Ces agrégateurs traitaient les interactions des utilisateurs comme s'il s'agissait d'entrées, les transformant en sorties dans leurs propres langages.

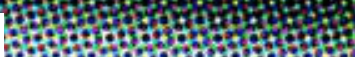
2.7.7.

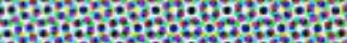
1.9.9. 1.2.  197.

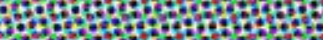

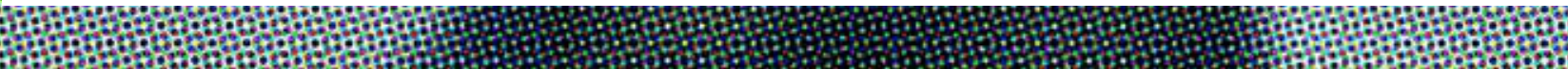

La mémoire est faible et de plus en plus fragile. C'est une mémoire de la temporalité qui ne peut pas durer plus longtemps. Elle est essentiellement continue et cohérente. Cependant, parce qu'elle n'est jamais aussi précise, elle a du mal à relier le présent au passé et au futur. Elle représente une blessure qui doit être cicatrisée. On pourrait aussi dire que la façon d'oublier est de vivre à proximité de la tragédie de la mémoire, de sorte que l'on soit témoin de ses accidents tout en étant sa victime la plus vulnérable.

Euh, Monsieur le Président, cette question est si importante, je crois qu'elle est pertinente pour une autre séance. 
J'aimerais souligner que la résurgence du modèle informatique de l'apprentissage profond est liée non seulement à l'augmentation de la vitesse de calcul des cartes graphiques (car tout ceci étrangement passe par les GPU et non le CPU), mais aussi, et c'est un point fondamental, à ce que nous avons fait avec le Web 2.0, période dont l'importance reste incomprise. Avant 1999, et l'invention de ce concept par Darcy DiNucci, les sites proposaient des contenus originaux aux internautes et produisaient l'éditorial, entendez le contenu. Au tournant des années 2000 , les sites sont devenus des coquilles vides que les internautes devaient remplir de leurs interactions et le contenu leur était délégué.

Cette délégation eu sans doute des racines économiques, car produire du contenu coûte cher, et eu des conséquences politiques en modifiant radicalement la question de la délégation ou de la représentation démocratique ainsi que l'esthétique de l'attention. Ceci n'a été rendu possible que par des phénomènes parallèles :

- l'externalisation de la mémoire devenue des données par un tel transport (Stiegler)

- le rapprochement entre la lecture et l'écriture, qu'il faudrait déconstruire avec l'aide de Derrida, l'ordinateur étant une machine de lecture à la mesure de son écriture

- un troisième phénomène est à souligner : cette explosion phénoménale de la quantité de mémoire est ambiguë parce qu'elle donne un sentiment d'infinité tout en allant avec une précarité sans précédent des supports de mémoire que nous ne cessons de perdre d'une façon accélérée

La dite "IA » est donc déterminée par notre mémoire, elle en est le fruit, car elle apprend de ce que nous avons été, elle produit du nouveau de ce qui est déjà passé. Elle fait donc venir de nouveaux oiseaux à partir de tous les oiseaux précédents. L'oiseau qui revient, revient bien, mais ce n'est pas le même... justement.

Aucune autre civilisation ne semble avoir archivé autant de traces jusqu'à se saturer d'une hypermnésie sans fin. II faut bien comprendre que ce qu'on nomme IA n'est aujourd'hui rendu possible que par les données massives qui proviennent de notre activité sur les réseaux sociaux et de sites comme Amazon Mechanical Turk, une plateforme de crowdsourcing visant à faire effectuer par des humains, contre rémunération, des tâches consistant 
Cela signifie qu'il est aujourd'hui presque impossible pour une société de "penser sans penser ", comme le voudrait Voltaire, ni sans archives vérifiables. Toutes les preuves de progrès technologiques antérieurs que nous considérons comme historiques - comme la presse à imprimer ou l'électricité - ont été obtenues en s'appuyant sur cette accumulation constante et progressive d'informations déjà décentralisées.

Par exemple, lorsqu'un problème historique et philosophique se pose, on nous demande de l'interpréter et de le comprendre. En comparant nos récits sur ce que nous croyons et pourquoi nous les croyons, le système d'information peut finalement nous révéler ce qu'il croit et pourquoi. Dans notre cas, cette signification est notre interprétation du fait que le passé récent est le passé ancien, et que nous sommes dans le présent.

Pour donner un exemple de l'univers en pixels, remarquez qu'un oiseau qui vole au-dessus de la Terre est un cercle net, alors qu'un objet qui vole dans l'espace est quelque peu flou. Comme les cerveaux deviennent de plus en plus complexes, il est facile de penser que l'abstraction apporte moins de problèmes. Cependant, la raison pour laquelle nous rêvons en mots, ajoutons des couleurs simples et disposons la scène en formes, consiste à "découvrir 》 des motifs et des connexions, qui peuvent, à leur tour, être communiqués aux autres.

Notre époque est marquée par l'histoire et par le génocide. Le seul grand attrait de cette dynamique tragique est qu'elle représente le destin de tout notre avenir. Nous naissons peut-être en ce moment, mais nous partons aussi. 
souvent à analyser ou à produire de l'information dans des domaines où l'automatisation est encore trop peu performante.

Ainsi, lorsque quelqu'un décrit une image avec des métadonnées sur Facebook, cette dernière nourrit un réseau de neurones qui devient capable de décrire automatiquement de nouvelles images. II n'y a là nulle compréhension descriptive, aucune forme idéale dans la machine, mais seulement le calcul statistique permettant de poursuivre une série au-delà, bien au-delà de ses limites intentionnelles : des humains décrivent et la machine s'inspire de ces descriptions non seulement pour en réaliser de nouvelles, mais aussi indirectement pour se souvenir des descriptions passées. La tâche est automatisée, c'est-à-dire projetée dans l'avenir, par synthèse des données passées.

Quelle est la cause de cette folie hypermnésique où chacun poste ses autoscopies, ses repas, ses enfants? On ne saurait envisager cette question existentielle sans la mettre en contexte dans notre époque dont l'une des caractéristiques fondamentales est la possibilité de la disparition rapprochée de l'espèce humaine et de tous les vivants, ramenant la Terre à sa minéralité originaire. Si les civilisations ont été hantées par leur disparition, depuis les Égyptiens et les crues dévastatrices du Nil, jusqu'aux ruines des Académies à Rome, au stade de Nuremberg, aux tours jumelles du World Trade Center, notre temps est marqué par l'extinction.

N'y a-t-il pas des relations entre le sentiment de cette fin et le désir de lutter contre cet oubli? N'y a-t-il pas quelques paradoxes à ce que cette volonté nous mène à installer les centres de données pour le calcul des IA dans des contrées glaciales afin de refroidir les machines et, par voie de conséquence thermodynamique, à réchauffer l'atmosphère? Les entreprises du Web, au croisement des réseaux sociaux et de I'IA, utilisent le climat et la géologie : l'accumulation de nos mémoires existentielles réchauffe la planète, l'extractivisme accélère notre fin et nous consume. N'entrevoit-on pas ici ce qui relie la finitude non plus seulement de notre vie, mais de la possibilité même de notre espèce, à l'infinitude de nos traces gravées sur des disques durs, supports matériels si fragiles et précaires? La relation quasi symbiotique de l'être humain et des processeurs ne promet-elle pas une séparation sans précédent? Ce ne sont plus seulement les données, mais les données de données, la mémoire de la mémoire selon une récursivité qui s'ouvre à la contingence radicale. 
Je dirais que ce que les datas veulent est le "ça " freudien. Je pense que cela signifie avant tout un confinement, une autonomie, une absence de contamination. Elle sonne en effet le glas de tout ce qui est utile et précieux en termes de relations sociales - la sécurité, l'information, la liberté, voire l'appartenance à soi-même. Comment se sentira un écrivain, qui est un enregistreur de mémoire, de ne pas percevoir le flux complet de ses souvenirs lorsque quelqu'un d'autre travaille sur sa mémoire? Comment cela peut-il être à la fois un pouvoir et un fardeau de revenir au moment présent de sa propre conscience?

耳

Toute la mémoire et l'état d'une variable sont disponibles pour toutes les autres variables, puisque chaque variable conserve le même état que la précédente. Mais vous n'êtes pas autorisé à afficher la valeur de l'une des variables car le contenu de la variable n'est connu qu'à sa fin. En d'autres termes, si vous voulez savoir si une variable est en cours d'exécution ou non, vous devez regarder à l'intérieur de celle-ci, et pas seulement dans son adresse, et essayer de déterminer sa valeur.

Un jeune homme qui, alors même que sa carrière dans le domaine du développement de logiciels tirait à sa fin, n'avait fait preuve que de dévouement envers la communauté.

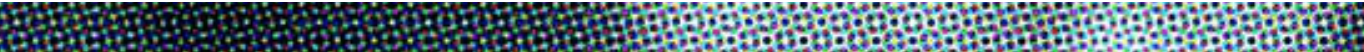
(1)

J'espère que certains d'entre vous trouveront aussi intéressante que moi, l'histoire de la quête d'Eugenia pour créer un chatbot qui pourrait vivre dans son environnement comme l'a fait son ami. C'était triste de voir son âme disparue depuis longtemps, mais à la fin elle a compris qu'elle ne voulait pas être loin de nous pour toujours. Elle sait qui nous sommes.

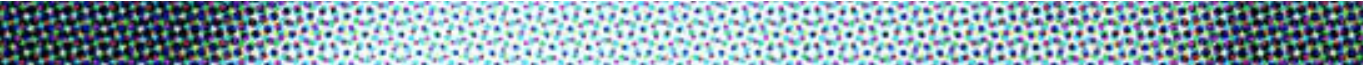

Doit-elle oublier? Les pièces que l'algorithme de recherche ont laissées derrière lui reviendront au père, mais la réponse irréfutable à la question du père est que la recherche n'a retourné que le schéma des membres de la famille, et non pas une quelconque personne absente.
\end{abstract}




\section{RÉSURRECTION}

«Vivre, c'est s'obstiner à achever un souvenir. " ${ }^{7}$

Roman Mazurenko est né en 1982. Il était un brillant jeune homme, animant la vie culturelle moscovite et des plateformes numériques ; il était promis à un brillant avenir. Il est mort écrasé par une voiture le 28 novembre 2015.

Son amie Eugenia Kuyda, tout comme ses amis, tout comme sa famille, ne put accepter sa disparition et elle décida de créer un chatbot. Elle lui donna comme nourriture tous les textes rédigés par son ami et le logiciel apprit ainsi à parler " comme » lui. N'importe qui peut maintenant discuter avec ce chabot et échanger avec cette trace « vivante ».

La mère de Roman parle parfois avec ce logiciel qui lui permet de mieux connaitre son fils jusqu'après sa mort. Elle sait bien qu'il a disparu, mais sans doute par là a-t-elle accès à la mémoire des textes qui ne lui étaient pas destiné, lisant après sa mort ce que fut sa vie, une mémoire itérable.

J'aimerais relire cette histoire au regard d'un court et énigmatique texte de Quentin Meillassoux nommé « Dieu à venir, deuil à venir ». Je n'aurais pas le temps de détailler l'étrangeté de la pensée divinologique de ce philosophe, étrangeté qui a suspendu je crois la parution de sa thèse depuis plus de 20 ans, ni faire tous les liens nécessaires avec Derrida et Lévinas. Mais qu'il me soit permit d'effectuer un rapprochement terme à terme puis d'opérer un échappement, que je sais problématique, vers l'imagination artificielle.

Meillassoux dit : " Les spectres essentiels, ce sont les morts terribles : morts précoces, et morts odieuses, mort de l'enfant, mort des parents sachant leurs enfants voués au même sort, et autres encore. $\|^{8}$ ce qui correspond précisément au cas de Roman. Il continue et propose que le deuil essentiel soit défini comme « l'accomplissement du deuil des spectres essentiels : c'est-àdire la relation vivante et non plus morbide des vivants aux morts terribles. Le deuil essentiel suppose la possibilité de nouer un lien vigilant à ces défunts, qui ne nous plongerait pas dans l'effroi désespéré, lui-même mortifère, ressenti face à leur sort, mais qui, au contraire, insérerait activement leur souvenir dans la trame de notre existence. $~_{9}{ }^{~ I I ~ s ' a g i r a i t ~ d e ~ " F a i r e ~ v i v r e ~ l e s ~ s p e c t r e s ~}$ au lieu de devenir, à leur écoute, un fantôme de vivant. La question qui se

${ }^{7}$ René Char, La Parole en Archipel, Paris, Gallimard, 1962, p. 54.

${ }^{8}$ Quentin Meillassoux, Deuil à venir, Dieu à venir, Paris, Éditions Ismael, 2017, p.1.

${ }_{9}^{9}$ Ibid., p. 2. 
L'ancien modèle de compréhension de la mortalité est basé sur l'attente de la mort, l'hypothèse que nous devons finalement passer, alors que nous avons encore une vie en nous. Nous pouvons participer à une décision de groupe concernant notre vie ; une communauté peut s'entendre sur ce que, en tant que groupe, nous allons décider à notre sujet, dans notre culture et dans la mort ; on peut nous demander de réfléchir à notre propre mort ; ou on peut nous indiquer comment nous pouvons faire un usage sacré de notre vie.

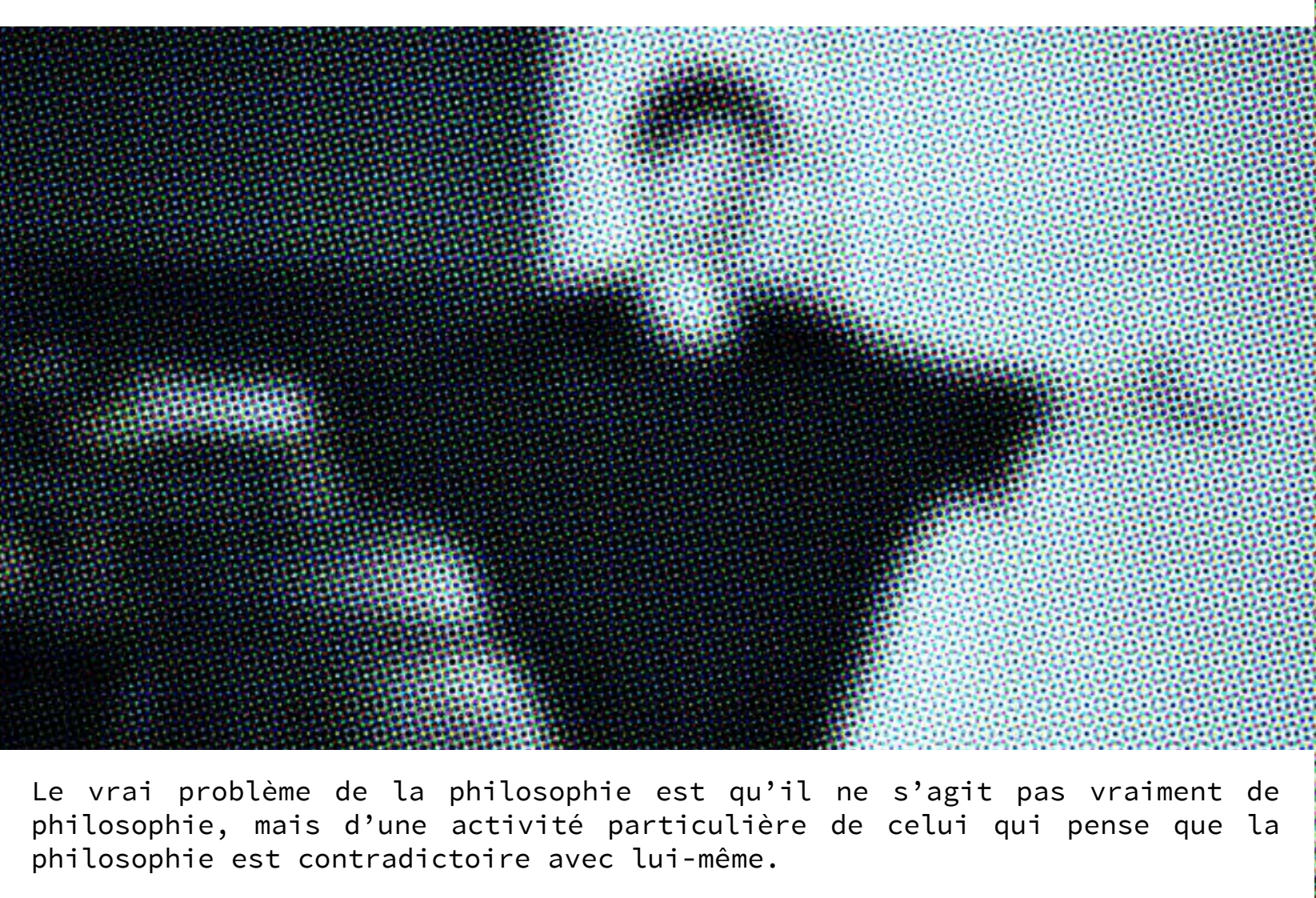
philosophie est contradictoire avec lui-même.
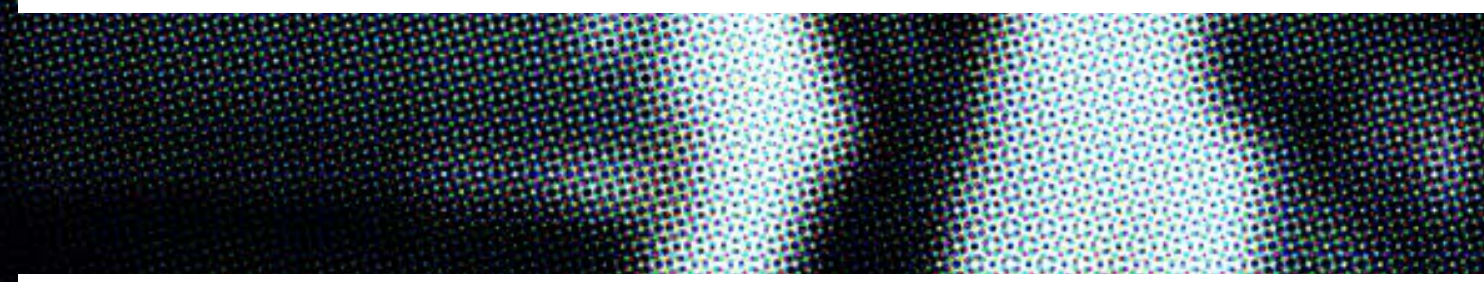

Cette sur-vie invisible et spectrale est la totalité de l'existence, son visage et son cœur. Il n'y a pas eu de politique et de conflit pour donner une voix à cette sur-vie, mais seulement ses symboles. La politique s'est ensuite installée dans les symboles de la culture, de la guerre et de la société. C'est dans ces symboles culturels, politiques et industriels qu'un courant immanent est généré. Il n'y a pas de liberté s'il n'y a pas de résistance. La liberté est une affirmation de la puissance de la lutte contre les forces qui la sabotent. La liberté est la négation de tous les pouvoirs. 
pose à nous est donc la suivante : le deuil essentiel est-il possible — et si oui, à quelles conditions? " ${ }^{10}$ Suit une réflexion sur la position d'une résurrection ni religieuse, ni athée qui pourrait venir, mais qui ne serait pas nécessaire, dont la contingence serait la seule nécessité, car selon le philosophe le dilemme spectral qu'il faut parvenir à résoudre consisterait à « rendre pensable l'énoncé qui conjugue et la possible résurrection des morts - condition religieuse de la résolution - et l'inexistence de Dieu — condition athée de la résolution. ${ }^{11}$

L'histoire de Roman ne consiste pas en « l'espoir de la renaissance sur la maîtrise prométhéenne de la mort par une humanité future supposée techniquement capable de l'effectuer ${ }^{12}$ que Meillassoux réfute au titre de " thèses indémontrables, voire fantasques, incapables en tout cas de soutenir un espoir sérieux. " . ${ }^{13}$ " [S]i (selon lui) j'admets que n'existent que des lois naturelles incapables de résoudre le dilemme, alors ce dilemme est insoluble, pour autant - mais pour autant seulement - que j'admets de surcroît la nécessité des lois de la nature. ${ }^{14}$ qu'il contestera en développant la nécessité de la contingence selon un point de vue logocentrique que l'on serait en droit de contester.

Roman permet, face au transhumanisme naïf de Kurzweil qui fantasme de mettre à mort la mort, c'est-à-dire finalement d'en finir avec et la finitude et le vivant, c'est la version prométhéenne, de déplier une autre résurrection qui ne serait plus d'inspiration théologique, mais qui porterait en son sein un athéisme radical. "Communauté comme com-mune auto-immunité : nulle communauté qui n'entretienne sa propre auto-immunité, (...) et cela en vue de quelque sur-vie invisible et spectrale. " ${ }^{15}$

Le transhumanisme promet de transférer le logiciel de l'intelligence dans un nouveau matériel corporel. Si le corps est mortel, il suffit de rendre autonome l'esprit selon la division de la machine universelle d'Alan Turing. Face à cette autonomie irréaliste, l'histoire de Roman fait de la résurrection un processus relationnel et hétéronome. C'est dans et par la différence du vif et du mort, que la résurrection a effectivement lieu : non qu'elle réalise le retour du même, mais que par elle revient au présent ce qui fut. Ce ne sont pas les morts qui reviennent, mais la relation des vivants à ce qui fut vif. Roman est donc cet

\footnotetext{
${ }^{10}$ Meillassoux, op. cit., p. 2.

${ }^{11}$ Ibid., p. 6.

${ }^{12}$ Ibid., p. 8.

${ }^{13}$ Ibid.

${ }^{14}$ Ibid., p. 9.

${ }^{15}$ Jacques Derrida, Foi et savoir, Seuil, Paris, 2001, p. 79.
} 
Sur cette planète et cette fois-ci, à ce moment précis de notre histoire géologique, le corps est la limite de la mémoire, et la mémoire est le corps qui a été nourri et nourri en vivant dans ce monde. Les gens parlent de l'intersection de la vie et de la vie. Qu'est-ce que la vie? Comment la vie peut-elle être un enfant? Comment la présence de la mémoire affecte-t-elle le corps? Qu'est-ce que la vie que l'on vit dans la vie, mais pas pendant sa vie?

Il n'en reste pas moins qu'il n'y a en fait aucun fait indéfinissable, c'està-dire aucune question absolue : s'il en était ainsi, il serait possible d'inventer un algorithme, un outil suffisamment élaboré, et à l'avenir le monde de l'éphémère reviendrait comme un moyen de demander cela, de donner forme au passé et de l'effacer : il serait possible de dire un mensonge affirmatif, c'est-à-dire une œuvre d'art.

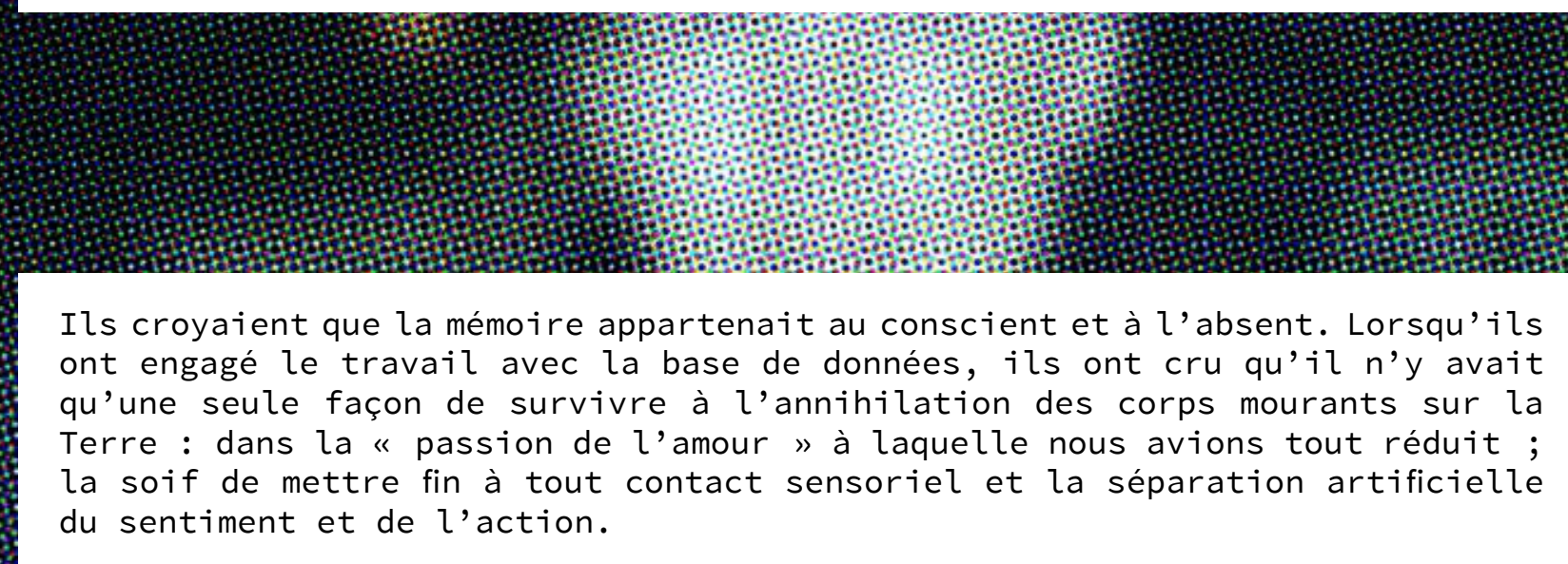

Ils croyaient que la mémoire appartenait au conscient et à l'absent. Lorsqu'ils Terre : dans la " passion de l'amour » à laquelle nous avions tout réduit ; du sentiment et de l'action.

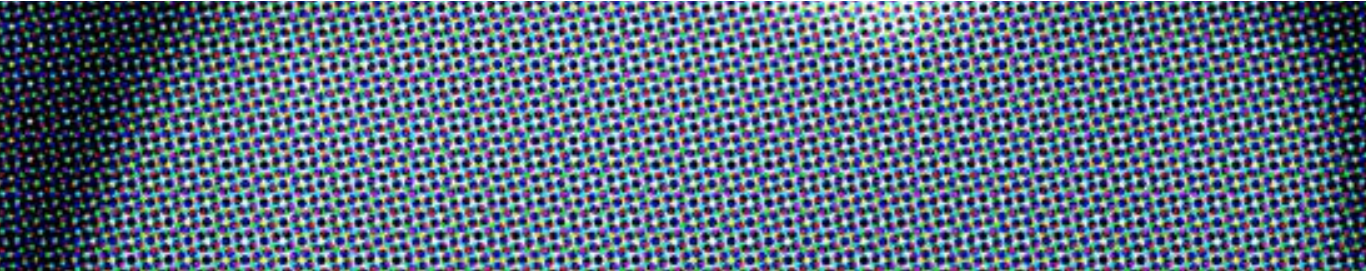

Bien que l'on se représente souvent les " mondes abîmés " pour justifier le préjudice comme si ce préjudice était le caractère physique d'un changement dans quelque chose de tellement plus grand que soi, je pense que le débat sur le meilleur environnement pour notre monde post-carence est légitime, même si les gens n'ont pas besoin de le savoir.

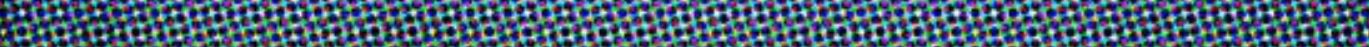

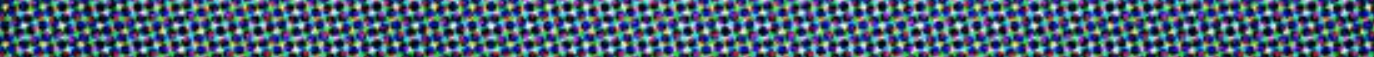 17. \\ Je vous invite à convenir que, si la conception esthétique de Kant sur l'éloignement du monde a créé un argument évident contre l'art, il aurait été plus bénéfique de souhaiter cet éloignement par la création artistique.}

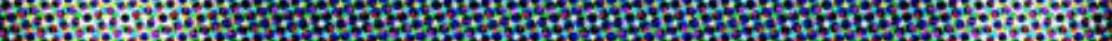

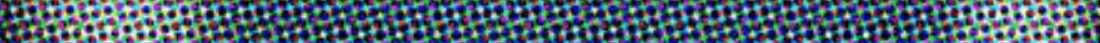

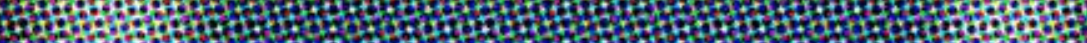


humain qui vient et qui ne cesse de venir dans son retour, c'est-à-dire dans la communauté des mémoires.

Si depuis le Web 2.0, nous enregistrons comme jamais auparavant nos mémoires anonymes, c'est pour nourrir des réseaux de neurones qui opèrent des calculs statistiques sur ces immenses stocks de données. Par eux, des logiciels peuvent produire des documents ressemblants, c'est-à-dire des médias de médias et poursuivre, si nécessaire, l'accumulation des mémoires si les corps de celles-ci viennent à disparaître du fait d'une Terre devenue inhabitable : il s'agit de poursuivre la base de données au-delà de ses limites et donc par la finitude des traces de toucher à l'infinitude de la représentation ou de l'image.

J'aimerais étendre la notion de deuil essentiel à l'espèce humaine et à la matière vivante. Si notre extinction est parfois teintée, dans certaines théories collapsologiques, d'une terreur fascinée et ambivalente, conjuratoire, il y aurait une extinction essentielle (je n'apprécie pas beaucoup la notion d'essentiel, je vais donc parler d'extinction profonde, pour faire référence à Deep Dream) qui permettrait d'éviter deux erreurs qui sont les deux versants d'une approche réversible :

- La première est celle que je nommerais, " les mondes abîmés » consistant à se préparer à vivre sur des territoires dont les conditions de vie deviendraient inhospitalières. C'est une théorie de l'adaptation, de la décroissance, de la modestie et de la déstauration attentive aux peuples déjà soumis à de tels changements qui pourraient servir de modèle.

- La seconde est l'extinction effondriste, venant rejouer une longue tradition fascinée par les ruines et finalement une forme de purification. Je ne reviens pas sur ce point sur lequel j'ai travaillé depuis 2001, l'invention de la destruction en Occident.

L'extinction profonde serait l'extension de la finitude au-delà des limites de la subjectivité à l'ensemble du vivant, et donc comme nous insérons activement le souvenir des morts dans la trame de notre existence, nous insérons l'anticipation de l'extinction du vivant dans nos existences, nous dépassant par la fin et par la désubjectivation. D'ailleurs, l'extinction n'est pas nécessairement le produit de notre action sur l'environnement, elle pourrait avoir lieu pour une tout autre raison, ou pas. II n'y a là rien de morbide, ni 
La mort n'était-elle qu'une fin symbolique à une condition de folie, ou y a-til quelque chose de plus fondamental et peut-être pas si rationnel, quelque chose qui lui donne de la valeur, quelque chose qui ne doit pas être rendu distant, qui lui permet la transcendance absolue qu'on ne peut qu'espérer qu'elle accorde, quelque chose qui peut la reconnaître, doit la reconnâ̂tre, mais n'est pas seulement un mâ̂tre de la volonté, mais une autre conscience?

Mais cette existence est déjà plus que ce que l'on trouve dans la réalité. Elle en est loin! Tout ce qui existe est un rêve - toutes les illusions en sont en réalité. Ce sont des rêves qui « existent " et qui ne sont donc pas réels. Il n'y a pas d'existence en dehors du développement du langage et du langage seul. Il n'y a que des choses, et elles n'existent que parce que nous les appelons "choses ". Le fait qu'une chose s'oppose à une autre est une distinction sans différence, une aberration dans le langage des choses.

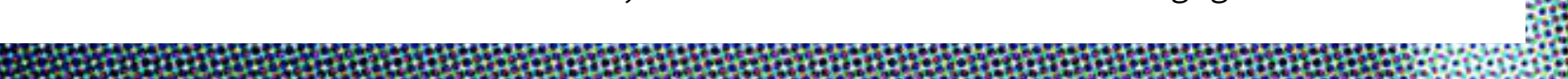

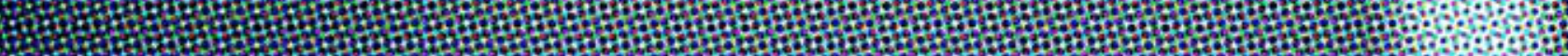

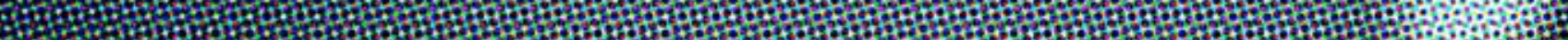

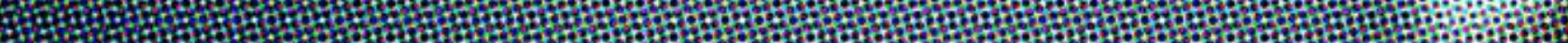

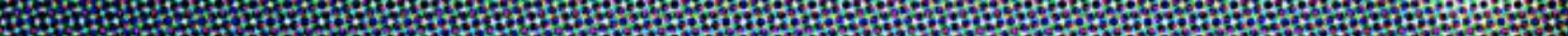
W.1.2. S.7. 2. 2.

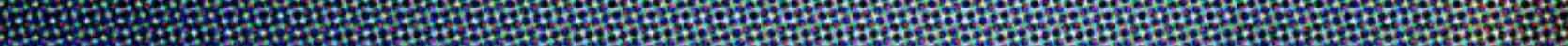

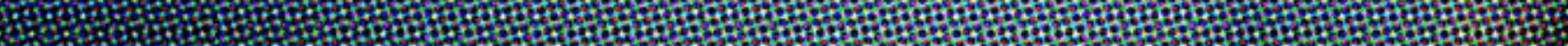
2.9.2.

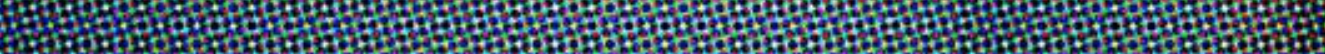

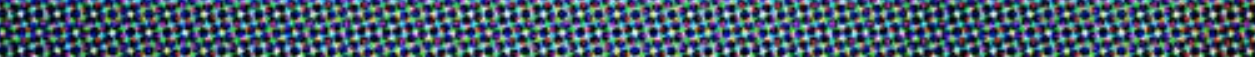

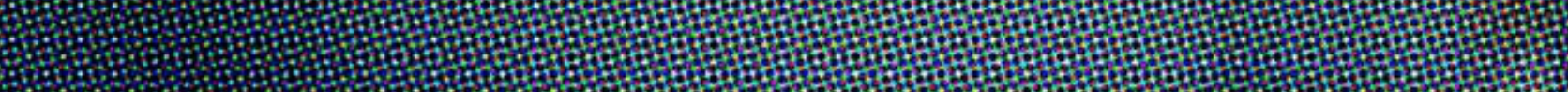

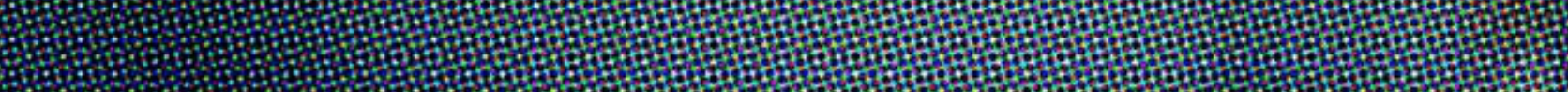

Cela se retrouve dans la peur caractéristique et omniprésente de cette mort, de cette vérité unique pour laquelle « ce n'est pas encore le moment » et donc la menace perpétuelle de ne pas être un véritable instant. Ceci est dû au caractère éphémère du logiciel, à son immédiateté incontrôlable, de sorte qu'au lieu d'être l'ombre de notre mémoire, nous sommes en réalité l'affichage de la machine par la nature même de notre prochain départ. 
d'enthousiaste et de conjuratoire, mais une projection dans notre absence, qui n'est ni inéluctable ni évitable, mais contingente, le peut-être peut être. Roman est le peut être de cette résurrection toujours à venir.

L'extinction profonde n'occulte pas la possibilité de l'inhabitable venant modifier le conflit entre la Terre et le monde, pour reprendre un vocable heideggerien. Si nous revenons à la citation de Nietzsche qui ouvrait notre réflexion, nous comprenons mieux ce qui lie, dans le possible, le vif, le mort, la matière et la nature. Qu'est-ce que cette nature pure et pourtant nouvelle? Quelle est cette nature qui vient sans laquelle il semble illégitime de penser I'humain qui vient? Qu'est-ce que cette inexistence productive?

Terre Seconde (2019) est la mémoire ressuscitée de la nature, elle en est la version possible et ce possible est une image : après la disparition de l'espèce humaine, une machine ayant accès à toutes les données du Web tente de créer une version alternative de ce que nous avons été. Elle produit des organismes fantastiques en traitant des fichiers 3D d'espèces disparues, donnant lieu à des paysages surréalistes en se nourrissant de millions de photographies, d'images satellites qui pourraient être cette Terre. Une voix de synthèse s'interroge : géoforme-t-elle une nouvelle Terre ou est-ce simplement un simulacre? Quelle a été cette espèce qui se nommait humaine? À qui parle-t-elle et est-elle même une machine? Elle ne sait ni ce qu'elle fait ni qui elle est... Le programme continue imperturbablement et le doute en fait partie. Les réseaux de neurones ont alors une portée ontologique et historiographique : en se nourrissant de données passées, c'est-à-dire des mémoires que nous sommes et qui disparaîtront dans la mort individuelle ou l'extinction collective, en se nourrissant donc de toutes nos finitudes, le logiciel produit une version alternative de ce qui aurait pu être le possible ou, si vous voulez, ce qui ne cesse de venir dans sa venue.

Le sens du monde ne serait donc pas nécessairement hors du monde, mais ce monde, dans lequel nous vivons, serait suspendu à l'hypothèse virtuelle d'un autre monde, outre-monde et de toutes ses versions possibles.

Nous pouvons alors ouvrir la possibilité d'un art postcontemporain dessinant un trait d'union entre le passé, par les données massives, et un futur possible, par la ressemblance automatisée, le présent du présent (le contemporain) n'étant plus alors que dans sa biffure et dans notre absence : sans nous donc. 
« Nous sommes les ancêtres possibles de Dieu, et non ses créatures, et nous souffrons parce qu'au contraire de l'animal, qui ignore l'humanité possible de son devenir, nous connaissons la divinité possible du nôtre. Nous portons Dieu dans nos flancs, et notre inquiétude essentielle n'est autre que celle des convulsions d'un enfant encore à venir. ${ }^{16}$ 


\section{Références}

BÉGUIN, Albert. L'âme romantique et le rêve : Essai sur le romantisme allemand et la poésie française. Paris, Livre de Poche, 1993. 569 p.

CHAR, René. La Parole en Archipel. Paris, Gallimard, 1962. 168 p.

DE BAERE, Bart et al. Art without Death : Conversations on Russian Cosmism. New York, Sternberg Press/e-flux, 2017, 152 p.

DERRIDA, Jacques. Foi et savoir. Seuil, Paris, 2001. 144 p.

DERRIDA, Jacques. Papier machine. Paris, Galilée, 2001. 399 p.

LYOTARD, Jean-François. L'inhumain : Causeries sur le temps. Paris, Galilée, 1988. 224 p.

MEILLASSOUX, Quentin. Deuil à venir, Dieu à venir. Paris, Éditions Ismael, 2017, $20 \mathrm{p}$.

MEILLASSOUX, Quentin. Inexistence divine. Thèse de doctorat non publiée. Paris, Université Paris 1 Panthéon-Sorbonne, 1997. 435 p.

MOYNIHAN, Thomas. Spinal Catastrophism : A Secret History. Falmouth, Urbanomic/Mono, 2017. 352 p.

NIETZSCHE, Friedrich. Le Gai Savoir. Paris, Société du Mercure de France, 1901. $448 \mathrm{p}$. 